\title{
Toward Biomorphic Control Using Custom aVLSI CPG Chips
}

\author{
M. Anthony Lewis \\ Iguana Robotics, Inc. \\ P.O. Box 628 \\ Mahomet, IL 61853 \\ tlewis@iguana-robotics.com \\ Avis H. Cohen \\ Dept. of Biology \\ Neuroscience and Cognitive Science \\ University of Maryland \\ College Park MD 20742 \\ ac61@umail.umd.edu
}

\author{
Ralph Etienne-Cummings \\ Dept of Electrical and Computer Engineering \\ Johns Hopkins University \\ Baltimore, MD 21218 \\ etienne@ece.jhu.edu \\ Mitra Hartmann \\ Division of Biology \\ California Institute of Technology \\ Pasadena, CA 91125 \\ hartmann@bbb.caltech.edu
}

\begin{abstract}
The locomotory controller for walking, running, swimming and flying animals is based on a Central Pattern Generator (CPG). Models of CPGs have been proposed and roboticists have adapted these models for the control of robots. These CPGs are modeled as systems of coupled non-linear oscillators. In this paper we describe the implementation of an adaptive CPG model in a compact, custom analog VLSI circuit. These circuits are very small, power efficient and inexpensive to manufacture in volume.

We demonstrate the function of the chip by controlling an underactuated, running robotics leg. This circuit has adaptive properties which allow it to tune its behavior based on sensory feedback. To our knowledge this is the first instance of an adaptive CPG chip.

This approach supports the construction of extremely inexpensive, low power and compact controllers for walking, flying and swimming machines.
\end{abstract}

\subsection{Introduction}

\subsection{Motivation}

Challenges for robotics in the future include the miniaturization of walking, running, and flying robots, increasing the real-time adaptability of robots to the environment, and the creation of mass-market consumer devices (e.g. Sony Dog [1]). These new technologies will require small, low-cost, power-efficient, and adaptive controllers, and may therefore benefit greatly from computational support, i.e. neuromorphic engineering, that is radically different than current microprocessorbased technology.

The basic principle of neuromorphic engineering is to use principles of biological information processing to address real-world problems. Using a neuromorphic approach, complete nervous systems can be built to control robots. These artificial nervous systems can be realized in very low cost, low power and low weight units.

It is well recognized that the physics of silicon is in many ways analogous to the biophysics of the nervous system [9]. Therefore, neuromorphic systems are often implemented in silicon using as much of the properties of device physics as possible. However the vast majority of work in neuromorphic engineering to date has concentrated on sensory processing (for example, the construction of silicon retinas [8] or silicon cochleas [10]).

In this paper we present a chip, based on established principles of the locomotor-control circuits in the nervous system, that mimics many of the features of a biological Central Pattern Generator (CPG). We show that the circuit, consuming less than one microwatt of power and occupying less than 0.4 square millimeters of chip area ${ }^{1}$ can generate the basic competence needed to control a robotics leg running on a circular treadmill. Furthermore, the circuit can use sensory feedback to stabilize in real time the rhythmic movements of the leg.

This technology can potentially provide inexpensive circuits that are adaptable, controllable and able to generate complex, coordinated movements. Secondly, such circuits could be used in miniature systems to modulate repetitive cyclical movements based on appropriate sensory feedback. These systems include miniature walking and running machines, and could also include miniature flapping and swimming machines currently under development.

\subsection{CPG Theory}

The basic notion of an autonomous neural circuit generating sustained oscillations was first articulated by Brown in the early part of this century [2]. The key idea is that an autonomous system of neurons can generate a rhythmic pattern of neuronal discharge that can drive muscles in a fashion similar to that seen during normal locomotion. Locomotor CPGs are autonomous in the sense that they can operate without input from higher centers or from sensors. Under normal conditions, however, these CPGs make extensive use of sensory feedback from the muscles and skin, as well as descending input [5]. Furthermore, the CPG transmits information upward to higher centers as well as to the periphery to modulate incoming sensory information.

\footnotetext{
${ }^{1}$ The actual real estate used by a circuit is dependent on the manufacturing technology. This circuit used 1.2micron technology. State of the art is currently 0.18 micron. Far smaller chips are possible
} 
The CPG is most often thought of as a collection of distributed elements. For example in the lamprey, a relatively simple fish-like animal, a small, isolated portion of the spinal cord can generate sustained oscillations. When the spinal cord is intact, these small elements coordinate their patterns of activity with their neighbors and over long distances ([3][7]).

It is well known that sensory input can modulate the activity of CPGs. Modulation of the CPG by sensory input can be seen quite clearly in the resetting of the phase of the CPG. For example, as a walking cat pushes its leg back, sensors in the leg muscles detect stretching. These sensors (called stretch receptors) signal this stretch to the nervous system. Their firing initiates the next phase of the CPG causing the leg to transition from stance to swing phase.

In the early 1980s Cohen and colleagues [4] introduced a model of the lamprey CPG using a system of phase-coupled oscillators. Later, a model called Adaptive Ring Rules (ARR), based on ideas in this earlier work was extended for use in robot control [11][12].

Here we demonstrate the implementation of an ARR in a silicon neural circuit. We will show it in autonomous operation and with sensory feedback from stretch receptors used to reset the CPG. We also demonstrate a property of our biomorphic leg: we show that our limb and its control circuit can not only produce stable rhythmic motion, but it can also compensate for intentional biases in the chip as well as mechanical complexity of an active hip and passive knee.

\subsection{Modeling CPGs on a Neuromorphic Chip}

CPGs are most often modeled as distributed systems of non-linear oscillators. In our implementation the basic coordination in the leg is achieved by phasically coupling two neurons together to achieve oscillations. When coupled together they are alternatively active. This alternating activity is the basic coordination needed to drive the hip of the robot. A phase control circuit governs the phase difference between the neurons.

These neurons drive two integrate-and-fire spiking motor neurons. These neurons are used to drive an actuator. The spiking neuron could also drive biological muscle or it could also be used to drive a pneumatic cylinder, a McKibben actuator or biomuscle directly.

In our experimental setup, the robot under control uses electric motors. To be compatible with this technology, it was necessary to low-pass filter the spiking neurons output to create a smooth graded signal

\subsection{Previous Work}

CPG chips and circuits have been created before. For example, Still reports on a VLSI implementation similar to a CPG circuit used to drive a small robot in [16][17]. This circuit captured some of the basic ideas of a CPG but did not incorporate a motor neuron output stage, and the system did not provide for adaptation via sensory input. However, she did demonstrate rudimentary control of a walking machine.
The work of DeWeerth and colleagues [13] captures the neural dynamics on a much more detailed level than has been achieved here. There are great difficulties in applying such a system to the control of a robot. Primarily, parameter sensitivity makes such circuits difficult to tune. To address this issue, DeWeerth et al. have implemented neurons that self-adapt their firing-rate [15]. The adaptation, however, is independent of external inputs from sensors. While detailed neural models are difficult to work with in silicon, we will undoubtedly learn a great deal from these efforts in the future.

Ryckebusch and colleagues [14] created a VLSI CPG chip based on observations in the thoracic circuits controlling locomotion in locusts. The resulting VLSI chip was used as a fast simulation tool to explore understanding of the biological system. Their system did not use feedback from sensors, nor was it connected to a robotic system. However, again their objective, of modeling a particular biological circuit, was different than the objective described in this paper.

Our work differs from the previous work in several respects. Firstly, we allow adaptation based on sensory input. Adaptation is shown as a phase resetting of the CPG based on certain sensory triggers (see section 3.0). Secondly, our chip has short-term memory devices that allow adaptation of the output parameters. In addition, we make use of integrate-and-fire neurons for the output motor neurons. Our abstraction is at a higher level than other reported work ([13][15]). We believe that by using a higher level of abstraction we will be able to more easily implement on-chip learning. In systems based on numerous inter-related parameters, it is not apparent how learning at the level of behavior can be coupled to low level parameter changes.

\subsection{The CPG Chip}

The CPG chip is designed to provide biologically plausible circuits for controlling motor systems. The chip contains electronic analogues of biological neurons, synapses and time-constants. In addition, the chip also contains dynamic analog memories, and phase modulators. Using these components, non-linear oscillators, based on the central pattern generators of biological organisms, can be constructed.

The dynamical properties of the neural circuits can also be adapted using direct sensory information. In this first version of the chip, shown in Fig. 1, all the components are individually accessible such that they can be connected with off-chip wiring to realize any desired circuit. In future versions, tested neural CPG circuits will be integrated with completely hardwired or programmable circuits.

\subsection{Hardware Components}

\subsubsection{The Neuron}

Our neuron uses an integrate-and-fire model. A capacitor, representing the membrane capacitance of biological neurons, integrates impinging charge. When the "membrane-potential" exceeds the threshold of a 


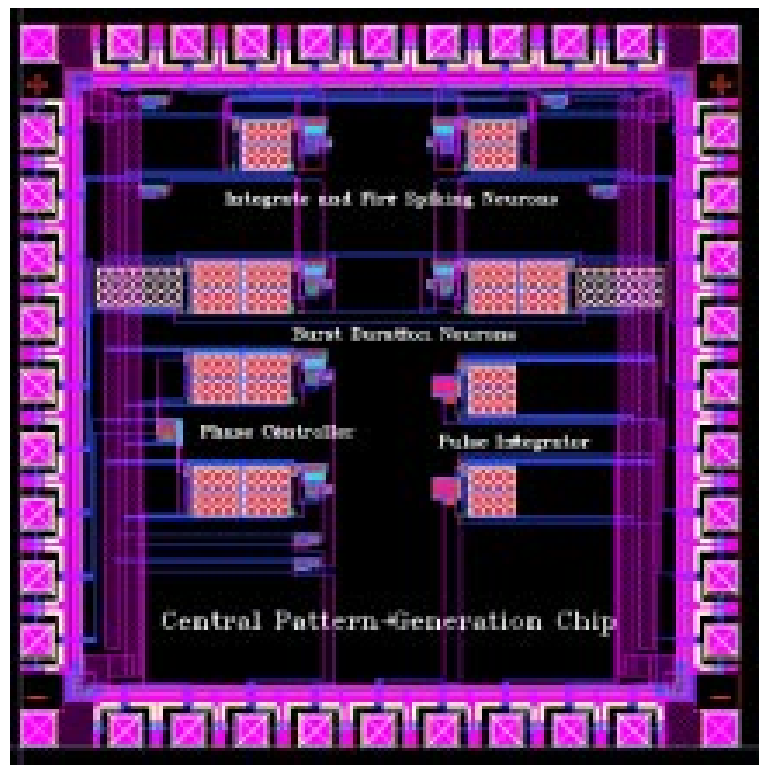

Figure 1. Layout of the CPG chip. Each component is wired to pins to facilitate the prototyping of oscillator circuits.

hysteretic comparator, the neuron outputs high. This logic high triggers a strong discharge current that resets the membrane potential to below the threshold of the comparator, thus causing the neuron output to reset. The process then starts anew. Fig. 2 shows a schematic of the neuron circuit.

Our neurons carry activation information in the frequency of spikes. The rate at which the membrane potential charges up controls the firing frequency of the neuron. This rate is given by the sum of the total charge flowing in and out of the membrane capacitance. The strength of the reset current source determines the width of each neural spike. The discharge current is usually set to a large value so that each spike is narrow and is not influenced by the charge injected onto the membrane capacitor. Typically, the neuron is set such that it fires at a nominal rate at rest; additional inputs increase or decrease the firing rate. Shunting inhibition can also silence the neuron. Equation 1 gives the dynamic equation for the neuron, where $S_{j}$ are the spike trains of input neurons, $I_{j}$ are the weights and $\mathrm{V}_{\mathrm{T}}$ are the thresholds of the comparator. The terms $I_{\text {spon }}$ and $I_{\text {dis }}$ set the spontaneous spike rate and spike duration of the neuron, respectively.

$$
\begin{aligned}
& C_{i}^{\text {mem }} \frac{d V_{i}^{\text {mem }}}{d t}=I_{\text {spon }}-S_{i} I_{\text {dis }}+\sum_{j} \bar{S}_{j} I_{j}^{+}-\sum_{j} S_{j} I_{j}^{-} \\
& S_{i}=\left\{\begin{array}{l}
1 \text { if } V_{i}^{\text {mem }}>V_{T}^{+} \\
0 \text { if } V_{i}^{\text {mem }}<V_{T}^{-}
\end{array} \quad(\text { b })\right.
\end{aligned}
$$

\subsubsection{The Synapse}

The synapse is simply implemented with current sources that charge or discharge the membrane capacitor. The spike trains impinging on a neuron activate switches that allow charge quanta to flow into or off the membrane capacitor. The amount of charge transferred per spike is

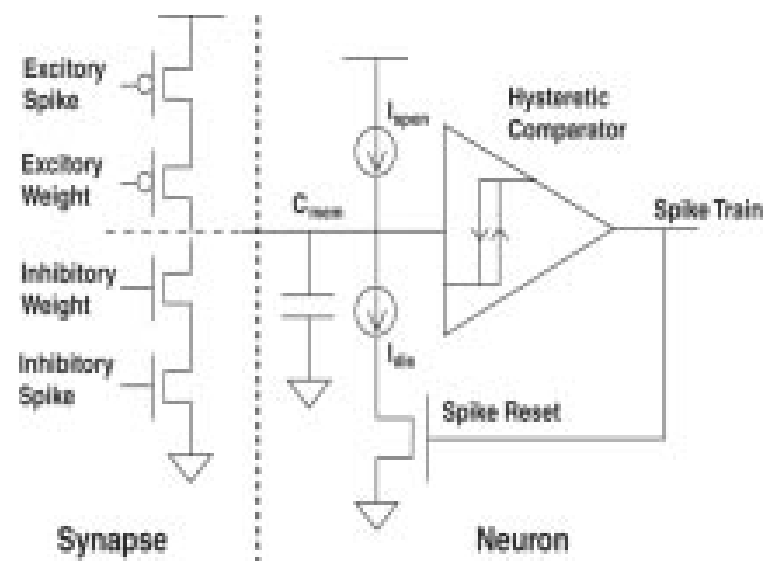

Figure 2. Schematic of the integrate-and-fire neuron and synapse.

the synaptic weight and is controlled by an applied voltage. Modulation of this voltage allows the adaptation of the neural firing rate and is used during learning. Fig. 2 shows the schematic of the synapse, while equation 1 shows how the neuron is affected by the synaptic weight.

\subsubsection{The Phase Controller}

To change the walking gait produced by a CPG, it is necessary to change the phase relationship between the two oscillators without affecting their frequency and burst duration. Typically this is realized by changing the coupling weights between the two oscillators. However this approach can affect the dynamics of the integrateand-fire neurons. Consequently, a special circuit is designed to accept the CPG signals and reproduce them with a controlled relative phase change. The phase controller circuit allows us to vary the phase relationship between the two sides of the CPG from 0 to 180 degrees by changing a control voltage. This mechanism also allows us to learn a particular phase relationship by adapting the control voltage in a closed loop fashion.

\subsection{The CPG Neural Circuit}

The neural circuits for creating the CPG are constructed using a cross-coupled square-wave oscillator and bursting motor neurons. Fig. 3 shows a schematic of this circuit. A master-slave configuration of the neurons allows us to construct an oscillator with a constant phase relationship. By setting the excitatory and inhibitory weights to equal values, a square-wave with a duty-cycle of $50 \%$ is obtained. The phase relationship between the two sides can be varied. The frequency of oscillation is set by the magnitude of the weights. This asymmetrically cross-coupled oscillator serves as the basic CPG unit that can be modified according to the application. By injecting or removing charge from the membrane capacitors of the oscillator neurons, the properties of the CPG can be altered. Alternatively, the phase controller can be used to modify the phase relationship between the two oscillators. For the experiments described in this paper, a strict 180 degrees phase relationship is required. Hence an inverted version of one of the oscillators is used, as shown in Fig. 


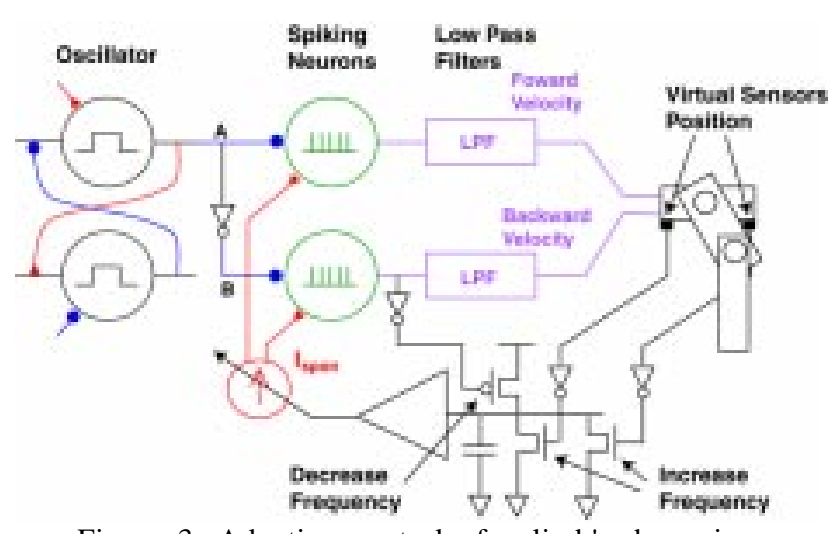

Figure 3. Adaptive control of a limb's dynamics using a neural CPG with learning capabilities.

3. Sensory inputs to the oscillators are used to stop or start the basic CPG unit.

The output of the basic CPG unit is used to inhibit the firing of the spiking (representing the motor) neurons. When the oscillator output is high, the motor neuron is not allowed to fire. This produces two streams of 180 degrees out of phase spike trains. These trains can be lowpass filtered to get a voltage which can be interpreted as a motor velocity. Consequently, the oscillator controls the length of the motor spike train, while the spike frequency indicates the motor velocity. These two attributes of the signal can be controlled independently to adjust the amplitude and speed of the swing of a limb, for example.

\subsection{CPG with Sensory Adaptation and Learning}

As shown in Fig. 3, the CPG neurons can be stopped or started with direct inhibitory and excitatory sensory inputs, respectively. When the inputs are received as strong inhibition, the membrane capacitor will be shunted and discharged completely. It will remain in this state until the inhibition is released, then the normal dynamics of the oscillator will continue from the inactive state. On the other hand, if the sensory input is received as a strong excitation, the oscillator will be driven into an active state. When the excitation is released, the oscillator will continue from the active state. Clearly, the charge-up or discharge of the membrane capacitor will be influenced by any direct sensory input. If the sensory inputs are periodic, the oscillator outputs can be driven such that they are phase locked to the inputs.

If learning is required, the chip provides a short-term (on the order of seconds) analog memory to store a learned weight. Clearly, this architecture favors continuous learning rule. Spikes from the motor neurons are used to increase or decrease a voltage on a capacitor; the voltage is used to set the connection weight of another neuron. In the absence of the training inputs, the stored weights decay at approximately $0.1 \mathrm{~V} / \mathrm{s}$. Fig. 3 shows a schematic for adapting the spiking frequency of the motor neurons based on the swing amplitude of the limb.

In Fig. 3, the limb is driven back and forth with a velocity signal that is obtained by low-pass filtering the activity of the motor neurons. Since the duration and frequency of the spike train is fixed by the CPG oscillator, changing the spiking frequency of the motor neuron alters the amplitude of the velocity signals, which in turn varies the swing amplitude of the limb. If the amplitude of swing does not reach the maximum positions, the motor neuron spike rate is increased. An increase in spike rate is kept bounded by negative feedback to the learning circuit. When the swing amplitude reaches maximum, the positive input to the learning circuit is reduced, thus allowing the spiking rate to settle to a constant value. The continuous negative feedback of the spike rate and the input from the position detectors maintain the learned spiking rate. The duration of the burst component of the spike train can be further controlled by feeding the position signals directly to the CPG oscillators to reverse the trajectory of motion at the end points. This allows very asymmetric forward and backward velocity signals to be adaptively re-centered, as shown in section 5 .

\subsection{Experimental Setup}

The experimental setup consists of a small robotic leg, the CPG chip, necessary components to interface the chip to the robotic leg, and data collection facility.

The robotic leg is a small (4" in height) two-joint mechanism. In our setup, only the top link is driven. The bottom link (the knee) is completely passive. The knee swings freely, rotating on a low friction ball-bearing joint. A hard mechanical stop prevents the knee from hyperextending.

The neurons of the CPG chip were interfaced to a servo motor using a rudimentary muscle model. The muscle dynamics are simulated as a low pass filter to smooth the output of the spiking neurons. This is followed by a pure integrator implemented in software. A bias was intentionally introduced into the chip to cause an asymmetry in the backward and forward swing of the leg. This bias might be typical of uncompensated parameters in a chip.

The robotic leg has three sensors on it. Two LVDT sensors monitor the position of the knee and hip joints. LVDT sensors are used because they introduced minimal friction and had infinite resolution. Additionally, the robot has a foot sensor to monitor ground contact forces.

The output of the hip LVDT is sampled digitally.

The signal is interval coded. Two intervals are selected as representing the extremes of movement of the hip (called virtual sensors position in Fig 3.). When these extremes are reached, the corresponding interval is active. This interval then sends a signal to the CPG chip causing an appropriate reset.

\subsection{Experiments}

\subsection{Running with a passive knee}

In this experimental setup, the CPG circuit drives the actuator in the hip joint. The knee joint is passive and rotates with very little friction.

The assembly is suspended above a rotating drum. The CPG circuit is started and the bursting rate is adjusted 


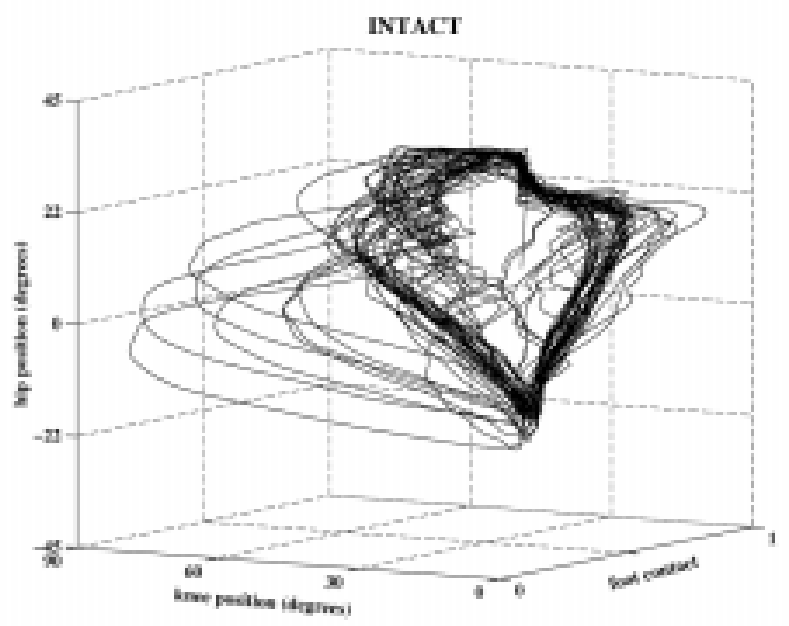

Figure 4. Hip, knee and foot-contact phase diagram. Most of the trajectory is in a tight bundle, while the outlying trajectories represent perturbations.

to approximately 2-3 $\mathrm{Hz}$. This rate had previously been found to produce a good running movement.

Data is collected for three sensors: Foot pressure, knee and hip. "Stretch receptor" sensory feedback from the hip is used as feedback to the CPG.

\subsection{Sensory feedback lesioning}

This experimental setup is similar to the first experiment. The difference is that sensor feedback is lesioned (turned off) periodically. We collect data as before.

\subsection{Results}

\subsection{Running Results with a Passive Knee}

A remarkable feature of this system is that the knee joint adapts the correct dynamics to enable running (!). As the upper limb swings forward, the lower limb rotates so that the foot comes off the ground. When the upper limb is suddenly accelerated backward, the momentum in the lower limb forces the knee to lock in place. At just the correct moment, the foot contacts the ground and the subsequent loading keeps the knee joint locked in place. As the foot travels backward it eventually begins to unload. Stored energy in the elastic foot causes it to 'kick up' and smartly snap off the ground, an effect most noticeable at higher velocities.

Figure 4 shows a phase plot of the knee, foot and hip position and foot contact. The bulk of the trajectory describes a tight 'spinning top' shaped trajectory while the few outlying trajectories are caused by disturbances. After a disturbance the trajectory quickly returns to its nominal orbit and we can infer that that the system is stable.

\subsection{Lesion Results}

Next we lesioned the sensory feedback to the leg periodically. Figure 5 shows the effect of lesioning on the position of the hip and knee joints as well as the tactile input to the foot. After lesioning the leg drifts backward

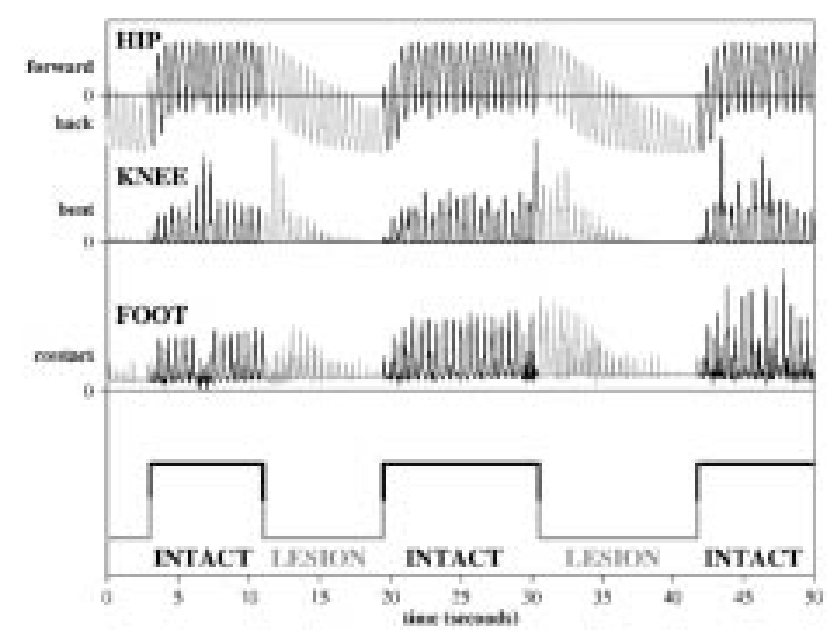

Figure 5. This figure shows the effect of lesioning sensory feedback. When the feedback is lesioned (Time 11-29 seconds and 31 to 42 seconds), the hip drives backward significantly. As it does the foot begins to lose contact with surface and the knee stops moving. When the lesion is reversed at 29 and 43 seconds, the regularity of the gait is restored.

significantly due to a bias built into the chip. When the sensory input is restored, the leg returns to a stable gait.

\subsection{Gait Stability}

Perturbations to the leg cause momentary disturbances. As seen above in Fig. 4, several of the trajectories are clear "outlyers" to the typical orbit, and result from environmental disturbances.

We found that sensory feedback could compensate for both the bias of the chip and environmental perturbations. Figure 6 shows restoration to a nominal orbit after perturbation in intact and lesioned cases. In the intact case, a perturbation at cycle ' 2 ' leads to outlying trajectories, but the trajectory is quickly restored to the nominal orbit. In the lesioned case, removal of sensory feedback causes the chip bias to destroy the trajectory of the leg. The gait quickly deteriorates.

\subsection{Summary and Conclusions}

In this paper we present the first experimental results of an adaptive VLSI neural chip controlling a robotic leg.

Basic rhythmic movements in animals are generated by a network of neurons in the spinal cord called the Central Pattern Generator or CPG. CPGs have been studied extensively and are beginning to be better understood. A model of the CPG was proposed by Cohen in the early 1980s and subsequently this CPG model was then adapted for use in robotic work [11][12].

In this paper we present a hardware implementation of this CPG model. Our custom VLSI chip, having only 4 neurons and occupying less than 0.4 square $\mathrm{mm}$ has the basic features needed to control a leg running on a treadmill.

The second point made in this paper is that running is a dynamic process. As has been noted by the biological 


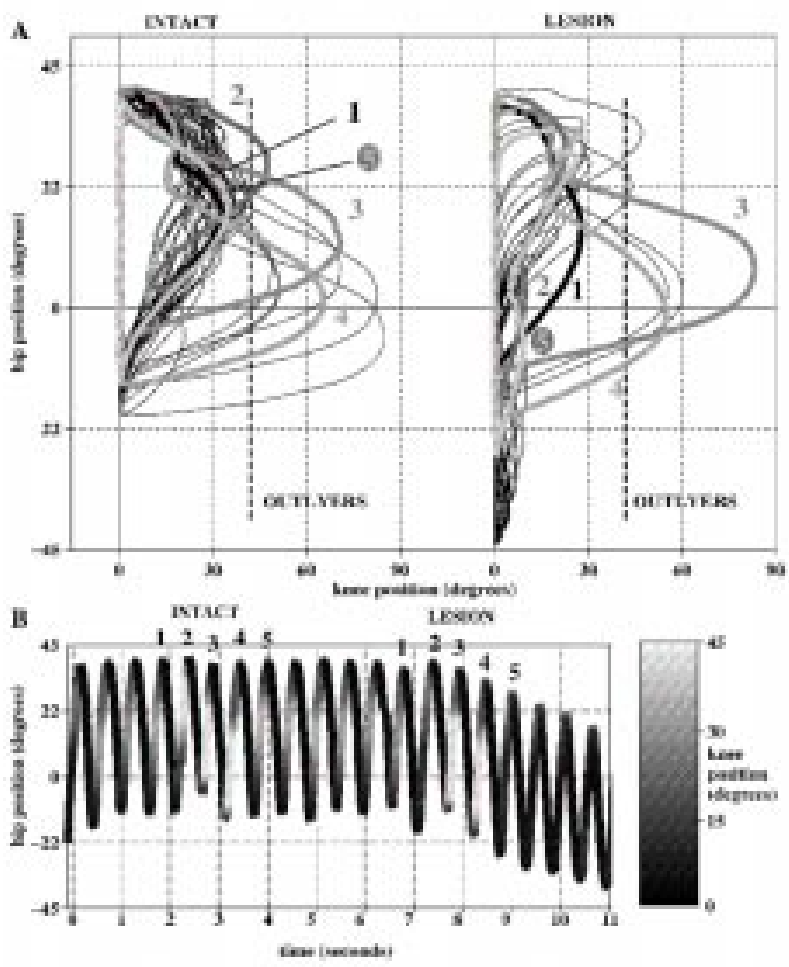

Figure 6. Effect of perturbations on gait with intact and lesioned sensory feedback. (A) Five sequential trajectories (numbered) in intact and lesioned conditions are represented as ranging between black and light gray. A perturbation at 2 in the intact case leads initially to worse trajectories ( 3 and 4 ), but quickly stabilizes to the nominal orbit (5). In the lesioned case, chip bias causes a perturbation at 2 from which the gait can not recover; the hip is forced backward (3,4, and 5). (B)The same ten trajectories shown in A presented as hip position through time, with knee position color-coded. Intact sensory feedback permits recovery while lesioning causes drift of both the hip and knee.

community (for example in the work of Robert Full [6]) as well as in the robotics community, much of the "intelligence" in running is actually in the dynamics. This is clearly illustrated in the current work by the use of an under-actuated robotic leg. In the results presented here, the energy injected into the hip is sufficient to excite an orbital trajectory of the knee as well. The hip, knee, and foot sensor orbit appears remarkably stable when the CPG circuit is stabilized using sensory feedback.

We conclude that the control of a running leg using a CPG chip is possible. We demonstrate that, at least in this experimental setup, running is possible using an underactuated leg. Finally, we demonstrate a basic adaptive property of phase resetting using a stretch receptor.

It should be emphasized that the system being controlled is non-linear and the chip itself uses non-linear elements to control it. We have a coupled system of nonlinear elements. We make no attempt to linearize the system. Instead we take advantage of the non-linearities.

Because (1) we do not make use of models, or linearization, (2) we adapt principles from biological systems, and (3) these principles can easily be implemented with low-power integrated circuits, we are able to achieve a very compact solution. Further experimentation with this system will allow us to determine if a robot can be made to walk by coupling together circuits as presented here. The current results, however, are promising.

\section{Acknowledgements}

We would like to acknowledge support of Grant No. N00014-99-0984 from ONR to Lewis and EtienneCummings and NIH grant MH44809 to Cohen.

\section{References}

[1] http://www.world.sony.com/robot/index.html

[2] Brown,T.G. (1914). On the nature of the fundamental activity of the nervous centres; together with an analysis of the conditioning of the rhythmic activity in progression, and a theory of the evolution of function in the nervous system. J. Physiol., Vol. 48, pp. 18-46.

[3] Cohen, A. H. and Wallén, P. (1980). The neuronal correlate to locomotion in fish: "Fictive swimming" induced in an in vitro preparation of the lamprey spinal cord. Exp. Brain Res. Vol. 41, pp. 11-18.

[4] Cohen, A. H., Holmes, P. J. and Rand, R. H. (1982). The nature of the coupling between segmental oscillators of the lamprey spinal generator for locomotion: A mathematical model. J. Math. Biol. Vol. 13, pp. 345-369.

[5] Cohen, A. H., Rossignol, S. and Grillner, S. (1988). Neural Control of Rhythmic Movements in Vertebrates. Wiley \& Sons.

[6] Full, R. J. (1993). Integration of individual leg dynamics with whole body movement in arthropod locomotion, In. R. D. Beer, R. E. Ritzmann, and T. McKenna, Eds, Biological Neural Networks in Invertebrate Neuroethology and Robotics, Academic Press, Inc, Boston.

[7] Grillner, S. and Wallén, P. (1985). Central pattern generators for locomotion, with special reference to vertebrates. Ann. Rev. Neurosci. Vol. 8, pp. 233-61.

[8] Koch, C., and Li, H., (Eds.) (1995). Vision Chips: Implementing Vision Algorithms with Analog VLSI Circuits, IEEE Computer Press.

[9] Mead, C., (Ed.) (1989).Analog VLSI and Neural Systems, Addison-Wesley, Reading, MA.

[10] Lazzaro, J., Wawrzynek, J., and Karmer, A. (1994), Systems Technologies for Silicon Audition, In: IEEE Micro, Vol 14, No. 3, pp. 7-15.

[11] Lewis, M.A.(1999). Sensory mediated gait adaptation in a quadruped robot, Autonomous Robots, (accepted).

[12] Lewis, M.A. (1996). Self-organization of Locomotory Controllers in Robots and Animals, Ph.D. Dissertation, Department of Electrical Engineering, University of Southern California, Los Angeles.

[13] Patel, G., Holleman, J. and DeWeerth, S. (1998). Analog VLSI Model of Intersegmental Coordination with NearestNeighbor Coupling. In Adv. Neural Information Processing, Vol. 10, pp. 791-725.

[14] Ryckebusch, S. , Wehr, M. and Laurent, G. (1994). Distinct Rhythmic Locomotor Patterns Can be Generated by a Simple Adaptive Neural Circuit: Biology, Simulation and VLSI Implementation, J. of Comp. Neuro, Vol 1, pp 339-358, 1994.

[15] Simoni, M., and DeWeerth, S. (1999). Adaptation in a VLSI Model of a Neuron. In: Trans. Circuits and Systems II, Vol. 46, No. 7, pp. 967-970.

[16] Still, S.(1998). Presentation at Neurobots Worshop, NIPS*98, Breckenridge, CO, USA.

[17] Still, S. and Tilden, M.W. (1998). Controller for a four legged walking machine, In: Neuromorphic Systems: 
Engineering Silicon from Neurobiology, Eds: L. S. Smith and A. Hamilton, World Scientific: Singapore, pp 138-148. 